\title{
EGFR NP_005219.2:p.K29_N298insG
}

National Cancer Institute

\section{Source}

National Cancer Institute. EGFR NP 005219.2:p.K29 N298insG. NCI Thesaurus. Code C101140.

A deletion of 268 amino acids of the epidermal growth factor receptor protein from the valine at position 30 through the arginine at position 297 followed by the insertion of a glycine between the lysine at position 29 and the asparagine at position 298. 